\title{
Association between matrix-metalloproteinase polymorphisms and prostate cancer risk: a meta-analysis and systematic review
}

This article was published in the following Dove Press journal: Cancer Management and Research

\author{
Hongxing Zhou' \\ Xuming Zhu ${ }^{2}$ \\ 'Department of Clinical Laboratory, \\ Changzhou Second Hospital affiliated \\ to Nanjing Medical University, \\ Changzhou, Jiangsu Province, China; \\ ${ }^{2}$ Department of Clinical Laboratory, \\ Wuxi People's Hospital affiliated to \\ Nanjing Medical University, Wuxi, \\ Jiangsu Province, China
}

Background: Data from published articles on the relationship between MMP polymorphisms and prostate cancer risk are conflicted and inconclusive, so a meta-analysis and systematic review were performed to assess the relationship.

Methods: Relevant research articles were identified from databases using a search strategy. Studies with the same MMP polymorphisms that could be quantitatively synthesized were included in the meta-analysis. Five comparison models (homozygote, heterozygote, dominant, recessive, and additive) were applied, and a subgroup analysis by case-group sample type was performed. Studies with different polymorphisms that could not be quantitatively synthesized were included in the systematic review.

Results: Eleven articles encompassing 22 studies involving 12 MMP polymorphisms were included in this paper. Among the studies included, 13 studies involving MMP1 rs1799750, MMP2 rs243865, and MMP7 rs11568818 were quantitatively synthesized for meta-analysis, and the other nine studies involving nine polymorphisms (MMP2 rs2285053, MMP2 rs1477017, MMP2 rs17301608, MMP2 rs11639960, MMP3 11715A/6A, MMP3 1161A/G, MMP3 5356A/G, MMP9 rs17576, and MMP13 rs2252070) were included in the systematic review. Meta-analysis showed no associations between MMP1 rs1799750, MMP2 rs243865, or MMP7 rs 11568818 and prostate cancer risk overall. Subgroup analysis by case-group sample type confirmed that no associations existed. The systematic review suggested that MMP3 11715A/6A and MMP9 rs17576 were associated with prostate cancer risk.

Conclusion: MMP polymorphisms are not associated with prostate cancer risk, except for MMP3 11715A/6A and MMP9 rs17576. However, it is necessary to conduct larger-scale, high-quality studies in future.

Keywords: matrix metalloproteinase, polymorphism, prostate cancer, meta-analysis

\section{Introduction}

A complex disorder resulting from the combined effects of multiple environmental and genetic factors, prostate cancer is the second-leading cause of cancer death in men. ${ }^{1}$ The underlying etiology of prostate cancer is still poorly understood. Genome-wide association studies on the genetic etiology of cancer have discovered some heritability of different cancer types. ${ }^{2}$ Single-nucleotide substitution, a kind of alteration in genetic sequence, can lead to cancer formation through biologically regulating a handful of molecular activities. ${ }^{3}$

A family of zinc endopeptidases, MMPs can cleave nearly all components of the extracellular matrix, as well as many other soluble and cell-associated proteins. ${ }^{4} \mathrm{MMPs}$
Correspondence: Xuming Zhu

Department of Clinical Laboratory, Wux People's Hospital Affiliated to Nanjing Medical University, 299 Qingyang Road, Wuxi, Jiangsu 214023, China

Tel +86I505223 3796

Email 7091159@qq.com 
play important roles in cancer development, invasion, and metastasis. ${ }^{5}$ At the genetic level, a number of studies have been carried out to assess associations between polymorphisms of MMPs and prostate cancer risk, ${ }^{6-14}$ but conclusions have been conflicting and inconclusive. For example, Srivastava et al found the MMP2 rs243865 polymorphism contributed to prostate cancer susceptibility, ${ }^{10}$ while Adabi et al found no association between MMP2 rs243865 polymorphism and prostate cancer risk. ${ }^{11}$ Individual studies with small samples may result in incorrect conclusions. Therefore, a comprehensive meta-analysis and systematic review were necessary to assess relationships between MMP polymorphisms and prostate cancer risk precisely.

\section{Methods}

\section{Search strategy}

The entire process of this meta-analysis and systematic review followed the guidelines of the PRISMA (preferred reporting items for systematic reviews and meta-analyses) statement (Table S1). ${ }^{15}$ The databases PubMed, Embase, and Web of Knowledge were searched with the following search terms: (MMP OR MMPs OR matrix metalloproteinase OR matrix metalloproteinases) AND (polymorphism OR polymorphisms OR single nucleotide polymorphism OR single nucleotide polymorphisms) AND (prostate cancer OR prostate carcinoma). The last search was on August 3, 2018. Additional published data were identified by reviewing references listed in each article. The literature search was performed by two investigators independently. Disagreement was resolved by discussion.

\section{Inclusion and exclusion criteria}

Inclusion criteria for this study were a focus on associations between MMP polymorphisms and prostate cancer risk, case-control design, available frequency of each genotype provided in both case and control groups to calculate OR and corresponding 95\% CI, and written in English. Exclusion criteria were reviews, editorials, comments, and animal studies and overlap with another included article.

\section{Data extraction}

Two investigators independently extracted author names, year of publication, country of origin, case-group sample type, source of control group, genotyping method, type of MMPs, names of polymorphisms, number of cases and controls, Hardy-Weinberg equilibrium (HWE) values, and frequency of genotypes. Consensus on extracted items was reached by discussion between the two investigators.

\section{Quality assessment}

The quality of each included study was assessed according to the quality-assessment criteria in Table S2. ${ }^{16}$ Quality scores of studies ranged from 0 to 15 , and studies with scores $\geq 9$ were regarded as being of high quality.

\section{Statistical analysis}

Meta-analysis was performed unless at least two studies concerning the same MMP polymorphism were included; otherwise, a systematic review was carried out. Pooled ORs and $95 \%$ CIs were calculated under five comparison models: homozygote, heterozygote, dominant, recessive, and additive. Pooled ORs assessed by $Z$-test were considered significant at $P<0.05$. HWE in the control group was checked by $\chi^{2}$ test, and disequilibrium was deemed present at $P<0.05$. Heterogeneity assumption was checked by a $\chi^{2}$-based $Q$-statistic test and quantified by $I^{2}$ values. If $I^{2}<50 \%$ or $Q$-test $P>0.10$, the -effect model was used. Otherwise, a random-effect model was used. Subgroup analysis by case-group sample type was also performed. Funnel plots and Egger's test were undertaken to examine publication bias. Publication bias was considered at $P<0.05$ for Egger's test. Statistical analyses for this paper were completed with Stata (College Station, TX, USA) version 12.0.

\section{Results}

\section{Literature search and study characteristics}

Figure 1 shows the selection process. A total of 26 articles were identified through the search strategy. ${ }^{6-14,17-33}$ Nine articles were removed based on the title or abstract, ${ }^{17-25}$ and

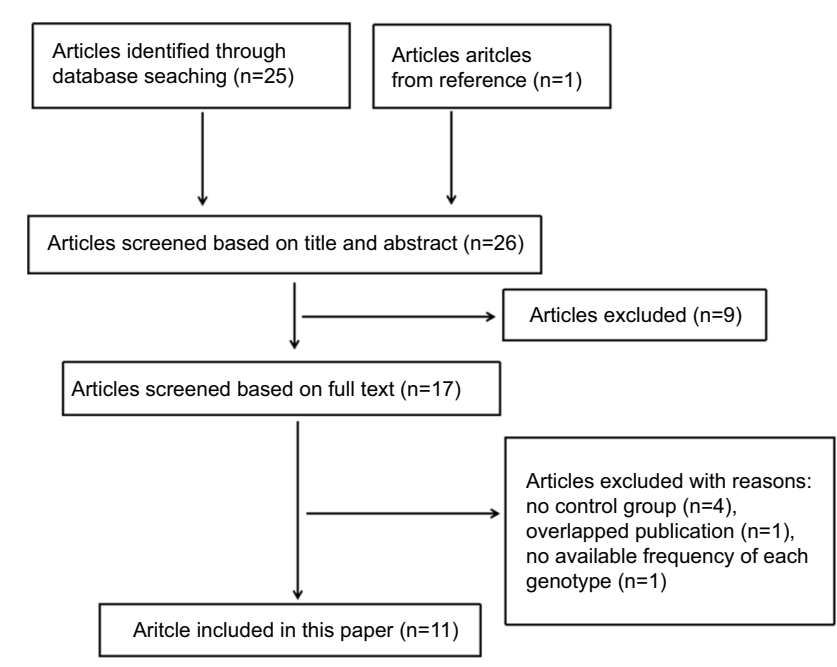

Figure I Flow diagram of study-selection process. 
the 17 remaining articles were screened for full text. Among these 17 articles, ${ }^{6-14,26-33}$ only eleven met inclusion criteria, because four did not have a control group, ${ }^{26-29}$ one overlapped with another, ${ }^{30}$ and one did not provide available frequency of each genotype in either the case group or control group. ${ }^{31}$ Ultimately, eleven articles encompassing 22 studies $^{6-14,32,33}$ and involving 12 polymorphisms were included in this paper. Their characteristics are listed in Table 1. Definitions of comparison models for the studies are listed in Table S3, and frequencies of genotypes from the meta-analysis and systematic review in Tables S4 and S5, respectively.

Among the included studies, 13 studies with three polymorphisms (five for MMP1 rs 1799750 involving 853 prostate cancer cases and 1,027 controls, six for MMP2 rs243865 involving 699 prostate cancer cases and 734 controls, and two for MMP7 rs11568818 involving 297 prostate cancer cases and 297 controls) were quantitatively synthesized for meta-analysis. ${ }^{6,8-10,12-14,32,33}$ The remaining nine studies with nine polymorphisms (MMP2 rs2285053, MMP2 rs1477017, MMP2 rs17301608, MMP2 rs11639960, MMP3 11715A/6A, MMP3 1161A/G, MMP3 5356A/G, MMP9 rs17576, and MMP13 rs2252070) involving 2,054 prostate cancer cases and 2,138 controls could not be quantitatively synthesized, and so the systematic review was performed. ${ }^{7,8,10,11,33}$

\section{Meta-analysis}

The results of meta-analysis for MMP1 rs1799750 (Table 2, Figure 2) showed that no significant associations were found in overall people (homozygote model, OR 1.16, 95\% CI 0.91-1.47, $P=0.237$; heterozygote model, OR 1.12, 95\% CI $0.94-1.33, P=0.223$; dominant model, OR $1.09,95 \%$ CI 0.94 $1.27, P=0.251$; recessive model, OR $1.09,95 \%$ CI $0.87-1.37$, $P=0.471$; additive model, OR 1.09, 95\% CI 0.97-1.23, $P=0.163$ ). When the studies were stratified according to blood samples of case groups (Table 2, Figure 2), no associations existed in any comparison model. Subgroups of tissue samples could not be assessed, because there was only one study included.

For the MMP2 rs243865 polymorphism (Table 3, Figure 3), meta-analysis showed no significant associations were found in people overall (homozygote model, OR 1.00, 95\% CI 0.84-1.20, $P=0.97$; heterozygote model, OR 1.08, 95\% CI 0.84-1.40, $P=0.54$; dominant model, OR 1.01, 95\% CI $0.87-1.18, P=0.875$; recessive model, OR $0.90,95 \% \mathrm{CI}$ $0.76-1.06, P=0.206$; additive model, OR 0.96 , 95\% CI 0.86 $1.08, P=0.521)$. Subgroup analysis by case-group sample type confirmed that no associations existed in any comparison model matter for blood or tissue samples (Table 3, Figure 3).
For MMP7 rs11568818 (Table 4, Figure 4), no significant associations were found in people overall (homozygote model, OR 0.95, 95\% CI 0.67-1.37, $P=0.796$; heterozygote model, OR 0.98 , 95\% CI 0.72-1.33, $P=0.908$; dominant model, OR 0.99 , 95\% CI $0.77-1.26, P=0.917$; recessive model, OR 0.91, 95\% CI 0.66-1.27, $P=0.592$; additive model, OR $0.97,95 \%$ CI $0.80-1.17, P=0.72$ ). Subgroup analysis by case-group sample type was not performed.

\section{Heterogeneity analysis}

For MMP1 rs1799750, MMP2 rs243865, and MMP7 rs11568818 polymorphisms, there was no obvious heterogeneity in any comparison model for people overall or for subgroup analyses (Tables 2-4).

\section{Publication-bias analysis}

For MMP1 rs1799750, funnel plots (Figure 5) and Egger's tests suggested no evidence of publication bias (homozygote model, $P=0.27$; heterozygote model, $P=0.187$; dominant model, $P=0.199$; recessive model, $P=0.351$; additive model, $P=0.226)$.

For MMP2 rs243865, funnel plots (Figure 6) and Egger's tests (homozygote model, $P=0.87$; heterozygote model, $P=0.864$; dominant model, $P=0.879$; recessive model, $P=0.826$; additive model, $P=0.927$ ) suggested no evidence of publication bias in the meta-analysis either.

For MMP7 rs11568818, publication-bias analysis was not conducted for the two studies involved.

\section{Systematic review}

In the systematic review (Table 5), two polymorphisms (MMP3 1171-5A/6A and MMP9 rs17576) were reported to be associated with prostate cancer risk, while another seven polymorphisms (MMP2 rs2285053, MMP2 rs1477017, MMP2 rs17301608, MMP2 rs11639960, MMP3 1161A/G, MMP3 5356A/G, and MMP13 rs2252070) were not associated with prostate cancer risk.

\section{Discussion}

Srivastava et al showed that MMP2 rs243865 polymorphism contributed to prostate cancer susceptibility, ${ }^{10}$ while Adabi et al showed no association between MMP2 rs243865 polymorphism and prostate cancer risk. ${ }^{13}$ Therefore, a comprehensive meta-analysis and systematic review were necessary. As a powerful tool for summarizing different studies, metaanalysis and systematic review refer to the use of statistical techniques to integrate results of included studies. ${ }^{15}$ 


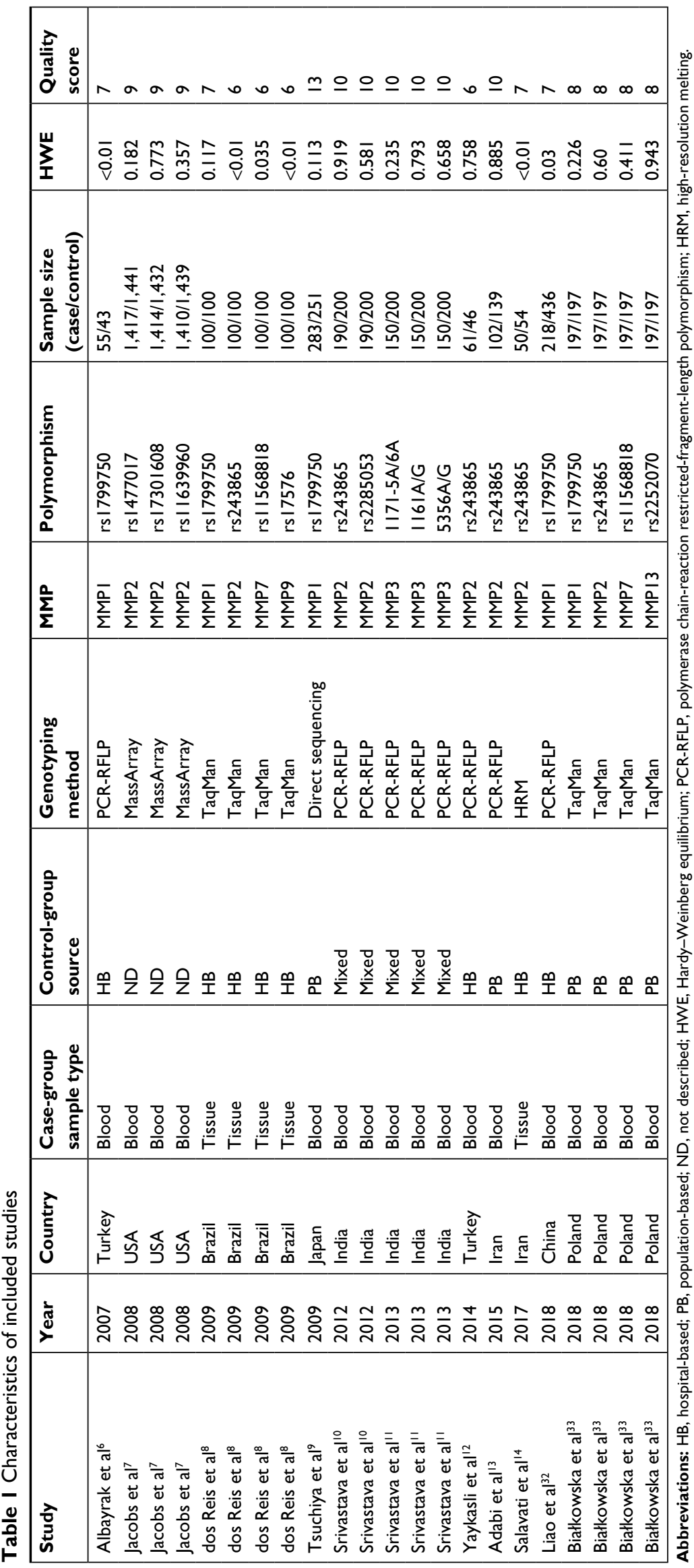


Table 2 Meta-analysis of association between MMPI rs I799750 and prostate cancer

\begin{tabular}{|c|c|c|c|c|c|c|}
\hline Comparison model & Subgroup & Studies & OR $(95 \% \mathrm{Cl})$ & $P_{O R}^{a}$ & $I^{2}(\%)$ & $P_{\text {het }} b^{b}$ \\
\hline \multirow[t]{2}{*}{ Homozygote } & Overall & 5 & $1.16(0.91-1.47)$ & 0.237 & 15.9 & 0.313 \\
\hline & Blood & 4 & $1.06(0.82-1.37)$ & 0.632 & 0 & 0.919 \\
\hline \multirow[t]{2}{*}{ Heterozygote } & Overall & 5 & $1.12(0.94-1.33)$ & 0.223 & 12.9 & 0.332 \\
\hline & Blood & 4 & $1.06(0.87-1.27)$ & 0.575 & 0 & 0.648 \\
\hline \multirow[t]{2}{*}{ Dominant } & Overall & 5 & $1.09(0.94-1.27)$ & 0.251 & 0.4 & 0.404 \\
\hline & Blood & 4 & $1.04(0.89-1.22)$ & 0.617 & 0 & 0.832 \\
\hline \multirow[t]{2}{*}{ Recessive } & Overall & 5 & $1.09(0.87-1.37)$ & 0.471 & 0 & 0.666 \\
\hline & Blood & 4 & $\mathrm{I} .03(0.8 \mathrm{I}-\mathrm{I} .3 \mathrm{I})$ & 0.818 & 0 & 0.982 \\
\hline \multirow[t]{2}{*}{ Additive } & Overall & 5 & $1.09(0.97-1.23)$ & 0.163 & 34.5 & 0.191 \\
\hline & Blood & 4 & $1.04(0.91-1.18)$ & 0.57 & 0 & $0.87 \mid$ \\
\hline
\end{tabular}

Notes: a ${ }^{P}$-value of $Z$-test for $O R$; ${ }^{b} P$-value of $Q$-test for heterogeneity.

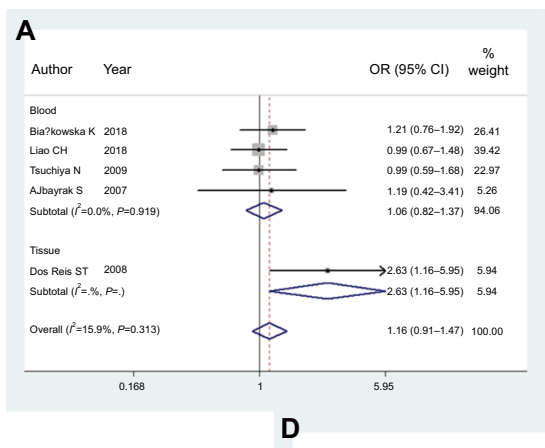

D

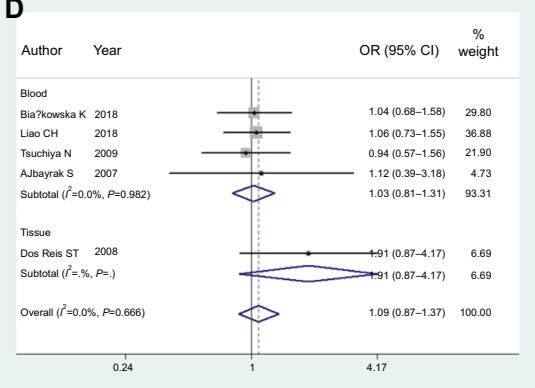

B

Author Year

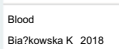

$\begin{array}{ll}\text { Bia? } 20 w s k a K & 2018 \\ \text { Lao CH } \quad 2018\end{array}$

Tsuchiya N 2009

A.Jbayrak S 2007

Tissue

Dos Reis ST 2008
Sublotal $\left(I^{2}=\%, P=\right.$.)

Overall $\left(f^{2}=12.9 \%, P=0.332\right)$
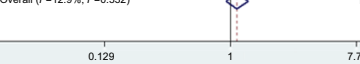

E

Author Year

Blood
Bia?kowstak 2018

Bia?kowska K 2018
$\begin{array}{ll}\text { Lia CH } \\ \text { CH }\end{array} 2018$

$\begin{array}{ll}\text { Tsuchiyan } & 2009 \\ \text { A Alayrak S } & 2007\end{array}$

Aubuytatal $\left(f^{2}=0.0 \%, P=0.871\right)$

Tissue
Dos Reis ST 2008

Dos Reils ST 2008
Subtotal $\left(r^{2}=\%, P=, 1\right.$

Overall $\left(l^{2}=34.5 \%, P=0.191\right)$

0.406

\section{C}

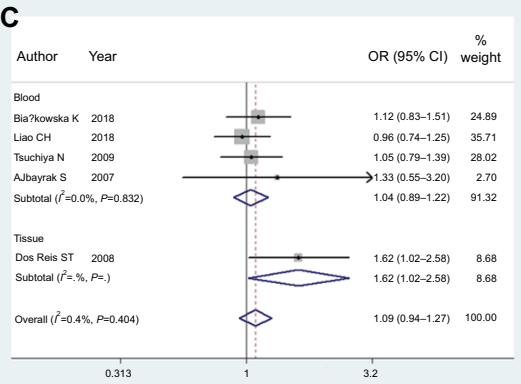

OR $(95 \%$ Cl) weight

$264(0.68-1.58) \quad 26.14$

$1.06(0.73-1.55) \quad 35.94$

$-1.12(0.39-3.3 .18) \quad 3.14$

$1.03(0.81-1.31) \quad 91.79$

\begin{tabular}{rr}
$\longrightarrow \quad 41(0.87-4.17)$ & 8.21 \\
\hline$\quad 91(0.87-4.17)$ & 8.21 \\
\hline
\end{tabular}

$1.09(0.87-1.37) \quad 100.00$

Figure 2 Forest plots of MMPI rs I 799750 and prostate cancer risk.

Notes: (A) Homozygote model; (B) heterozygote model; (C) dominant model; (D) recessive model; (E) additive model.

Table 3 Meta-analysis of association between MMP2 rs243865 and prostate cancer

\begin{tabular}{|c|c|c|c|c|c|c|}
\hline Comparison model & Subgroup & Studies & OR $(95 \% \mathrm{Cl})$ & $P_{O R}^{a}$ & $I^{2}(\%)$ & $P_{\text {het }}{ }^{b}$ \\
\hline \multirow[t]{3}{*}{ Homozygote } & Overall & 6 & $1.0(0.84-1.20)$ & 0.97 & 0 & 0.998 \\
\hline & Blood & 4 & $0.99(0.8 \mathrm{I}-\mathrm{I} .2 \mathrm{I})$ & 0.92 & 0 & 0.986 \\
\hline & Tissue & 2 & $1.06(0.7 \mid-1.56)$ & 0.787 & 0 & 0.825 \\
\hline \multirow[t]{3}{*}{ Heterozygote } & Overall & 6 & $1.08(0.84-1.40)$ & 0.54 & 0 & 0.894 \\
\hline & Blood & 4 & I.0I (0.76-I.34) & 0.967 & 0 & 0.972 \\
\hline & Tissue & 2 & $1.48(0.82-2.68)$ & 0.919 & 0 & 0.777 \\
\hline \multirow[t]{3}{*}{ Dominant } & Overall & 6 & $1.01(0.87-1.18)$ & 0.875 & 0 & 0.997 \\
\hline & Blood & 4 & $1.0(0.84-1.18)$ & 0.963 & 0 & 0.994 \\
\hline & Tissue & 2 & $1.08(0.77-1.50)$ & 0.66 & 0 & 0.778 \\
\hline \multirow[t]{3}{*}{ Recessive } & Overall & 6 & $0.9(0.76-1.06)$ & 0.206 & 0 & 0.957 \\
\hline & Blood & 4 & $0.91(0.75-1.09)$ & 0.305 & 0 & 0.801 \\
\hline & Tissue & 2 & $0.87(0.60-1.25)$ & 0.442 & 0 & 0.886 \\
\hline \multirow[t]{3}{*}{ Additive } & Overall & 6 & $0.96(0.86-1.08)$ & 0.521 & 0 & 0.987 \\
\hline & Blood & 4 & $0.96(0.85-1.09)$ & 0.511 & 0 & 0.892 \\
\hline & Tissue & 2 & $0.98(0.77-1.26)$ & 0.903 & 0 & 0.871 \\
\hline
\end{tabular}

Notes: a ${ }^{P}$-value of $Z$-test for $O R$; ${ }^{b} P$-value of $Q$-test for heterogeneity. 
A

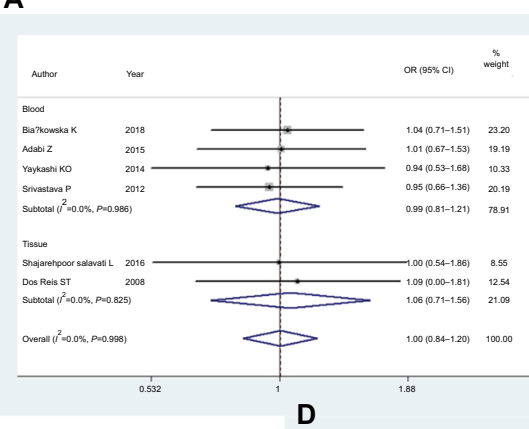

D
B

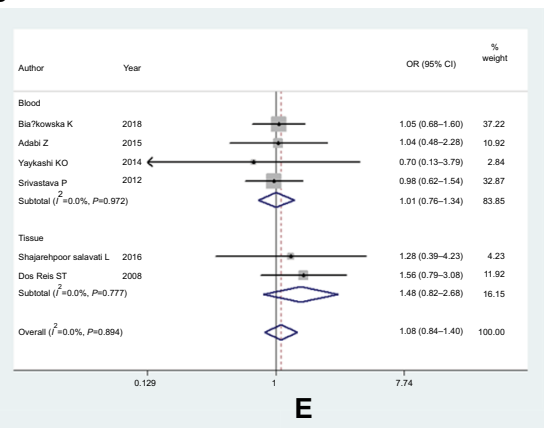

C

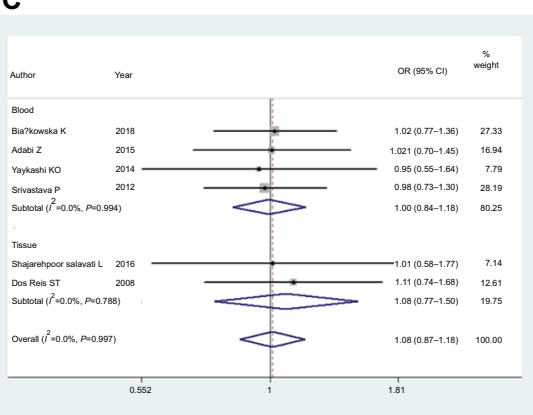

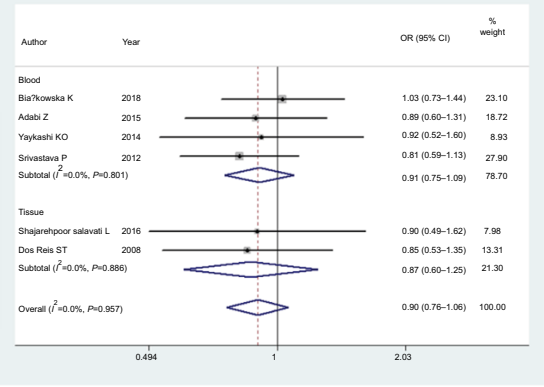

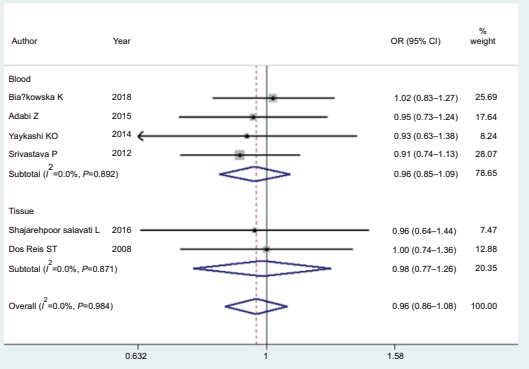

Figure 3 Forest plots of MMP2 rs243865 and prostate cancer risk.

Notes: (A) Homozygote model; (B) heterozygote model; (C) dominant model; (D) recessive model; (E) additive model.

Table 4 Meta-analysis of association between MMP7 rs I I5688I8 and prostate cancer

\begin{tabular}{|l|l|l|l|l|l|}
\hline Comparison model & Studies & OR $(\mathbf{9 5 \%} \mathbf{C l})$ & $\boldsymbol{P}_{\text {OR }}{ }^{\text {a }}$ & $\boldsymbol{I}^{\mathbf{2}}(\%)$ & $\boldsymbol{P}_{\text {het }}{ }^{\text {b }}$ \\
\hline Homozygote & 2 & $0.95(0.67-1.37)$ & 0.796 & 45.9 & 0.174 \\
Heterozygote & 2 & $0.98(0.72-1.33)$ & 0.908 & 0 & 0.435 \\
Dominant & 2 & $0.99(0.77-1.26)$ & 0.917 & 0 & 0.39 \\
Recessive & 2 & $0.91(0.66-1.27)$ & 0.592 & 53.7 & 0.142 \\
Additive & 2 & $0.97(0.80-1.17)$ & 0.72 & 56 & 0.132 \\
\hline
\end{tabular}

Notes: ${ }^{P}$-value of $Z$-test for $O R$; ${ }^{b}$-value of $Q$-test for heterogeneity.

A

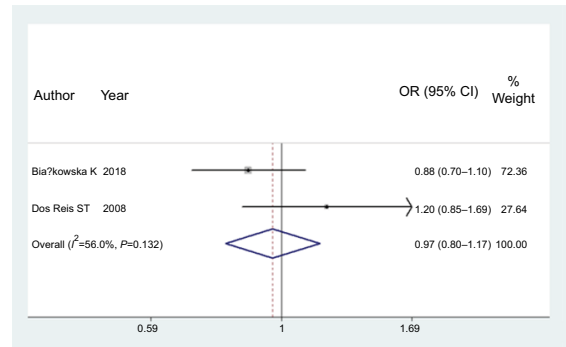

D

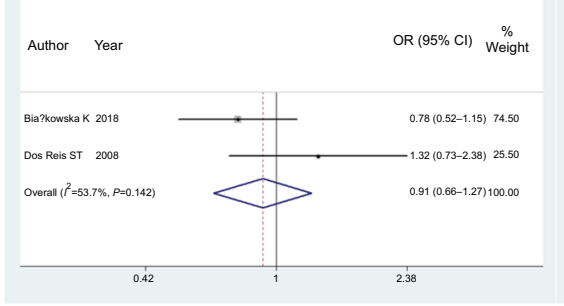

B

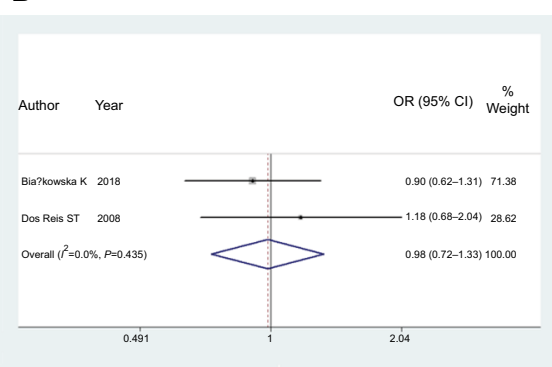

\section{E}

C

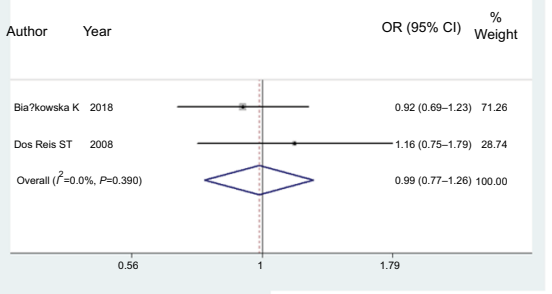

OR (95\% Cl) $\stackrel{\%}{\text { Weight }}$

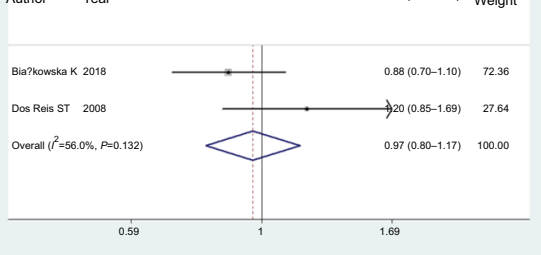

Figure 4 Forest plots of MMP7 rs I I5688 I8 and prostate cancer risk.

Notes: (A) Homozygote model; (B) heterozygote model; (C) dominant model; (D) recessive model; (E) additive model. 
A

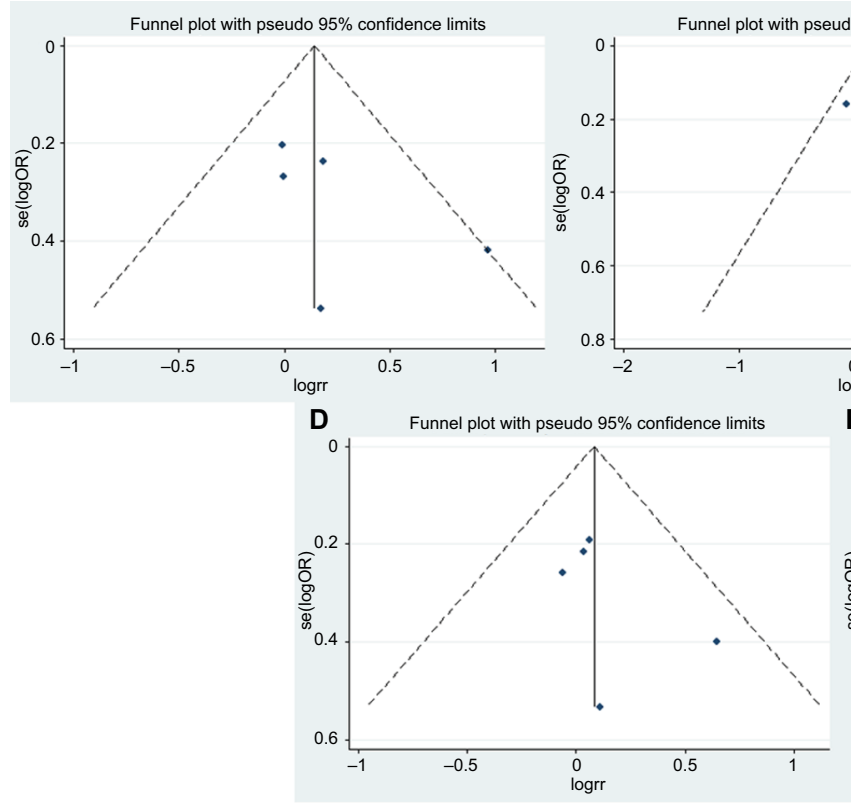

C

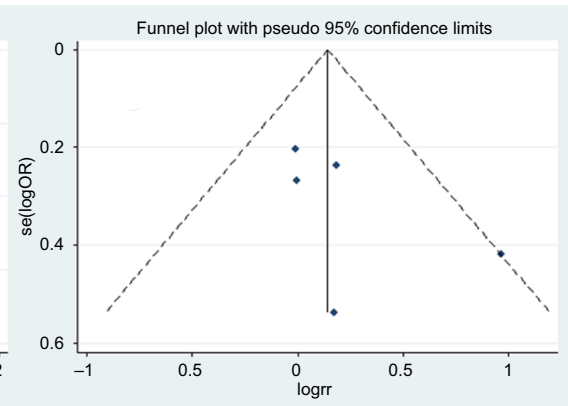

Figure 5 Funnel plots of MMPI rs I799750 and prostate cancer risk.

Notes: (A) Homozygote model; (B) heterozygote model; (C) dominant model; (D) recessive model; (E) additive model.

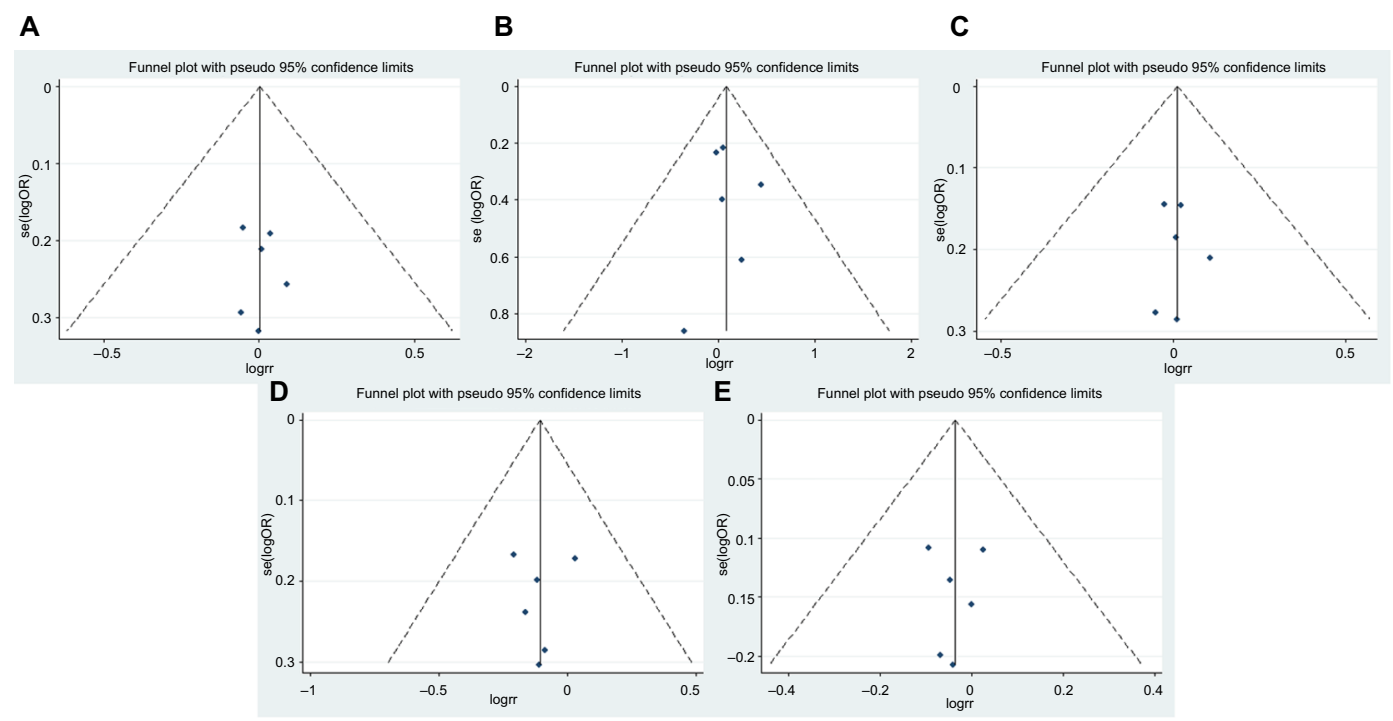

Figure 6 Funnel plots of MMP2 rs243865 and prostate cancer risk.

Notes: (A) Homozygote model; (B) heterozygote model; (C) dominant model; (D) recessive model; (E) additive model.

This meta-analysis of five studies for MMP1 rs1799750, six studies for MMP2 rs243865 and two studies for MMP7 rs11568818 demonstrated that MMP1 rs1799750, MMP2 rs243865 polymorphisms and MMP7 rs11568818 were not associated with prostate cancer. Subgroup analysis by case-group sample type confirmed that no associations existed in any comparison model. We attributed the negative conclusions of our meta-analysis to two factors: firstly, only articles in English were included, and thus other related articles failed to be included; and secondly, some lower-quality studies were included, resulting in unpersuasive conclusions.

Although this systematic review of nine studies involving nine polymorphisms revealed that MMP3 $11715 \mathrm{~A} / 6 \mathrm{~A}$ and MMP9 rs17576 were associated with prostate cancer risk, its conclusion needs more research to support it, because each polymorphism had only one study. MMP9 can produce prostate cancer indirectly via triggering TGF $\beta$ activation, because an increase in TGF $\beta$ signaling will lead to cancer development and progession. ${ }^{34,35}$ 
Table 5 Systematic review of association between MMPs polymorphisms and prostate cancer

\begin{tabular}{|c|c|c|c|c|c|c|c|}
\hline \multicolumn{8}{|c|}{ A Homozygote model, Heterozygote model, Dominant model } \\
\hline \multirow[t]{2}{*}{ MMP } & \multirow[t]{2}{*}{ SNP } & \multicolumn{2}{|l|}{ Homozygote model } & \multicolumn{2}{|c|}{ Heterozygote model } & \multicolumn{2}{|l|}{ Dominant model } \\
\hline & & OR(95\% Cl) & $\boldsymbol{P}$ & OR(95\% Cl) & $P$ & OR(95\% Cl) & $\boldsymbol{P}$ \\
\hline MMP2 & rs2285053 & $0.95(0.663-1.361)$ & 0.780 & $0.975(0.617-1.542)$ & 0.915 & $0.976(0.735-1.297)$ & 0.868 \\
\hline MMP2 & rs 1477017 & $0.937(0.807-1.089)$ & 0.398 & $0.974(0.842-1.128)$ & 0.726 & $0.975(0.876-1.086)$ & 0.646 \\
\hline MMP2 & rsl7301608 & $0.929(0.797-1.083)$ & 0.346 & $0.960(0.831-1.109)$ & 0.583 & $0.969(0.870-1.080)$ & 0.568 \\
\hline MMP2 & rsll1639960 & $0.958(0.827-1.111)$ & 0.573 & $0.994(0.857-1.153)$ & 0.933 & $0.986(0.886-1.098)$ & 0.802 \\
\hline MMP3 & $117 I-5 A / 6 A$ & $3.339(1.035-10.774)$ & 0.044 & $0.837(0.530-1.322)$ & 0.446 & $0.961(0.629-1.468)$ & 0.853 \\
\hline MMP3 & $1161 \mathrm{~A} / \mathrm{G}$ & $1.068(0.7 \mid 2-1.603)$ & 0.751 & $1.096(0.702-1.711)$ & 0.686 & $1.042(0.768-1.413)$ & 0.792 \\
\hline MMP3 & $5356 \mathrm{~A} / \mathrm{G}$ & $1.08 \mid(0.684-1.709)$ & 0.738 & I.I4 (0.763-I.706) & 0.522 & $1.064(0.782-I .447)$ & 0.695 \\
\hline MMP9 & rs 17576 & $0.025(0.002-0.242)$ & 0.001 & $0.444(0.28 \mathrm{I}-0.702)$ & 0.001 & $0.449(0.286-0.705)$ & 0.001 \\
\hline MMPI3 & rs 2252070 & $0.957(0.653-1.402)$ & 0.822 & $0.988(0.653-1.494)$ & 0.954 & $0.984(0.739-1.309)$ & 0.909 \\
\hline
\end{tabular}

\begin{tabular}{|c|c|c|c|c|c|}
\hline \multicolumn{6}{|c|}{ B Recessive model, Additive model } \\
\hline \multirow[t]{2}{*}{ MMP } & \multirow[t]{2}{*}{ SNP } & \multicolumn{2}{|l|}{ Recessive model } & \multicolumn{2}{|l|}{ Additive model } \\
\hline & & OR(95\% Cl) & $P$ & OR(95\% Cl) & $P$ \\
\hline MMP2 & rs2285053 & $0.812(0.585-1.125)$ & 0.21 & $0.91(0.735-1.125)$ & 0.383 \\
\hline MMP2 & rs 1477017 & $0.901(0.787-1.031)$ & 0.129 & $0.95(0.875-1.032)$ & 0.226 \\
\hline MMP2 & rs 17301608 & $0.913(0.796-1.048)$ & 0.195 & $0.95 I(0.875-1.034)$ & 0.238 \\
\hline MMP2 & rsll 639960 & $0.903(0.79 \mid-1.030)$ & 0.129 & $0.958(0.882-1.040)$ & 0.301 \\
\hline MMP3 & $117 I-5 A / 6 A$ & $3.667(I .|45-| I .74 I)$ & 0.029 & I.III (0.765-I.6I5) & 0.581 \\
\hline MMP3 & $116 \mathrm{IA} / \mathrm{G}$ & $0.997(0.693-1.433)$ & 0.986 & $\mathrm{I} .026(0.8 \mathrm{I} 4-\mathrm{I} .292)$ & 0.83 \\
\hline MMP3 & $5356 \mathrm{~A} / \mathrm{G}$ & $0.857(0.573-\mid .28 I)$ & 0.452 & $0.996(0.784-1.266)$ & 0.975 \\
\hline MMP9 & rs 17576 & $0.2(0.023-1.743)$ & 0.145 & $0.437(0.292-0.653)$ & 0.001 \\
\hline MMPI3 & rs2252070 & $0.885(0.628-\mid .247)$ & 0.484 & $0.948(0.763-1.177)$ & 0.627 \\
\hline
\end{tabular}

We noticed two previous meta-analyses had investigated the relationships of MMP1 rs1799750 or MMP2 rs243865 and prostate cancer risk. ${ }^{17,18}$ We read these carefully with great interest. Neither included other MMP polymorphisms, except for MMP1 rs1799750 and MMP2 rs243865. ${ }^{17,18}$ For MMP2 rs243865, our meta-analysis did not enroll the study by Jacobs et al, because it did not provide available frequency of genotypes. ${ }^{7}$ Conversely, both the previous meta-analyses included this study and thus concluded significant association. ${ }^{17,18}$ For MMP1 rs1799750, our paper enrolled two additional studies ${ }^{32,33}$ compared with one previous meta-analysis, ${ }^{17}$ and obtained a similar result. The major strengths of our paper lie in focusing on the relationship between MMP polymorphisms and prostate cancer risk comprehensively and systematically.

Some limitations still existed in our paper. First, several included studies contained small samples, which could lead to unpersuasive conclusions. Second, departure from HWE was detected in some studies. Third, there was a lack of a unified criterion for including studies.

\section{Conclusion}

In summary, our paper shows that MMP polymorphisms are not associated with prostate cancer risk, except for MMP3 1171-5A/6A and MMP9 rs 17576. However, it is necessary to conduct more large-scale and high-quality studies in future.

\section{Disclosure}

The authors report no conflicts of interest in this work.

\section{References}

1. Collak FK, Demir U, Ozkanli S, Kurum E, Zerk PE. Increased expression of YAP1 in prostate cancer correlates with extraprostatic extension. Cancer Biol Med. 2017;14(4):405-413.

2. Dai J, Shen W, Wen W, et al. Estimation of heritability for nine common cancers using data from genome-wide association studies in Chinese population. Int J Cancer. 2017;140(2):329-336.

3. Geng P, Li J, Wang N, et al. PSCA rs2294008 polymorphism with increased risk of cancer. PLoS One. 2015;10(8):e0136269.

4. Radisky ES, Raeeszadeh-Sarmazdeh M, Radisky DC. Therapeutic potential of matrix metalloproteinase inhibition in breast cancer. $J$ Cell Biochem. 2017;118(11):3531-3548.

5. Egeblad M, Werb Z. New functions for the matrix metalloproteinases in cancer progression. Nat Rev Cancer. 2002;2(3):161-174.

6. Albayrak S, Cangüven O, Göktaş C, Aydemir H, Köksal V. Role of MMP-1 $1 \mathrm{G} / 2 \mathrm{G}$ promoter gene polymorphism on the development of prostate cancer in the Turkish population. Urol Int. 2007;79(4):312-315.

7. Jacobs EJ, Hsing AW, Bain EB, et al. Polymorphisms in angiogenesisrelated genes and prostate cancer. Cancer Epidemiol Biomarkers Prev. 2008;17(4):972-977.

8. dos Reis ST, Pontes J, Villanova FE, et al. Genetic polymorphisms of matrix metalloproteinases: susceptibility and prognostic implications for prostate cancer. $J$ Urol. 2009;181(5):2320-2325.

9. Tsuchiya N, Narita S, Kumazawa T, et al. Clinical significance of a single nucleotide polymorphism and allelic imbalance of matrix metalloproteinase-1 promoter region in prostate cancer. Oncol Rep. 2009;22(3):493-439.

10. Srivastava P, Lone TA, Kapoor R, Mittal RD. Association of promoter polymorphisms in MMP2 and TIMP2 with prostate cancer susceptibility in North India. Arch Med Res. 2012;43(2):117-124. 
11. Srivastava P, Kapoor R, Mittal RD. Impact of MMP-3 and TIMP-3 gene polymorphisms on prostate cancer susceptibility in North Indian cohort. Gene. 2013;530(2):273-277.

12. Yaykaşli KO, Kayikçi MA, Yamak N, et al. Polymorphisms in MMP-2 and TIMP-2 in Turkish patients with prostate cancer. Turk J Med Sci. 2014;44(5):839-843.

13. Adabi Z, Mohsen Ziaei SA, Imani M, et al. Genetic polymorphism of MMP2 gene and susceptibility to prostate cancer. Arch Med Res. 2015;46(7):546-550.

14. Salavati LS, Tafvizi F, Manjili HK. The association between MMP2 $-1306 \mathrm{C}>\mathrm{T}$ (rs243865) polymorphism and risk of prostate cancer. Ir J Med Sci. 2017;186(1):103-111.

15. Moher D, Liberati A, Tetzlaff J, Altman DGPRISMA Group. Preferred reporting items for systematic reviews and meta-analyses: the PRISMA statement. Int J Surg. 2010;8(5):336-341.

16. Thakkinstian A, Mckay GJ, Mcevoy M, et al. Systematic review and meta-analysis of the association between complement component 3 and age-related macular degeneration: a HuGE review and meta-analysis. Am J Epidemiol. 2011;173(12):1365-1379.

17. Weng H, Zeng XT, Wang XH, Liu TZ, He DL. Genetic Association between Matrix Metalloproteinases Gene Polymorphisms and Risk of Prostate Cancer: A Meta-Analysis. Front Physiol. 2017;8:975.

18. Liu K, Gu S, Liu X, et al. The MMP2 rs243865 polymorphism increases the risk of prostate cancer: A meta-analysis. Oncotarget. 2017;8(42):72933-72938.

19. Haque S, Akhter N, Lohani M, Ali A, Mandal RK. Matrix metalloproteinase-2 -1306 C>T gene polymorphism is associated with reduced risk of cancer: a meta-analysis. Asian Pac J Cancer Prev. 2015;16(3):889-896.

20. Liu M, Zhao YY, Yang F, et al. Evidence for a role of GPRC6A in prostate cancer metastasis based on case-control and in vitro analyses. Eur Rev Med Pharmacol Sci. 2016;20(11):2235-2248.

21. Ben J, Jin G, Zhang Y, et al. Class A scavenger receptor deficiency exacerbates lung tumorigenesis by cultivating a procarcinogenic microenvironment in humans and mice. Am J Respir Crit Care Med. 2012;186(8):763-772.

22. Amankwah EK, Sellers TA, Park JY. Gene variants in the angiogenesis pathway and prostate cancer. Carcinogenesis. 2012;33(7): 1259-1269.

23. Nangia-Makker $P$, Wang Y, Raz T, et al. Cleavage of galectin-3 by matrix metalloproteases induces angiogenesis in breast cancer. Int J Cancer. 2010;127(11):2530-2541.
24. Ahirwar DK, Mittal RD. Re: Genetic polymorphisms of matrix metalloproteinases: susceptibility and prognostic implications for prostate cancer. S. T. dos Reis, J. Pontes, Jr., F. E. Villanova, P. M. D. Borra, A. A. Antunes, M. F. Dall'oglio, M. Srougi and K. R. M. Leite. J Urol 2009; 181: 2320-2325. J Urol. 2010;183(3):1258.

25. Jain S, Chakraborty G, Kundu GC. The crucial role of cyclooxygenase-2 in osteopontin-induced protein kinase $\mathrm{C}$ alpha/c-Src/IkappaB kinase alpha/beta-dependent prostate tumor progression and angiogenesis. Cancer Res. 2006;66(13):6638-6648.

26. Schveigert D, Valuckas KP, Kovalcis V, Ulys A, Chvatovic G, Didziapetriene J. Significance of MMP-9 expression and MMP-9 polymorphism in prostate cancer. Tumori. 2013;99(4):523-529.

27. Jaboin JJ, Hwang M, Lopater Z, et al. The matrix metalloproteinase-7 polymorphism rs 10895304 is associated with increased recurrence risk in patients with clinically localized prostate cancer. Int J Radiat Oncol Biol Phys. 2011;79(5):1330-1335.

28. Gil Ugarteburu R, Rivas del Fresno M, González Rodríguez I, et al. Matrix metalloproteinase- 9 polymorphisms in the diagnosis of prostate cancer. A preliminary experience. Arch Esp Urol. 2010;63(2): $125-132$.

29. dos Reis ST, Villanova FE, Andrade PM, et al. Matrix metalloproteinase-2 polymorphism is associated with prognosis in prostate cancer. Urol Oncol. 2010;28(6):624-627.

30. dos Reis ST, Villanova FE, de Andrade PM, et al. Polymorphisms of the matrix metalloproteinases associated with prostate cancer. Mol Med Rep. 2008;1(4):517-520.

31., Profile StudyAustralian Prostate Cancer BioResource (APCB)IMPACT Study, et al. Association analyses of more than 140,000 men identify 63 new prostate cancer susceptibility loci. Nat Genet. 2018;50(7):928-936.

32. Liao $\mathrm{CH}, \mathrm{Wu} \mathrm{HC}, \mathrm{Hu} \mathrm{PS}$, et al. The association of matrix metalloproteinase-1 Promoter polymorphisms with prostate cancer in taiwanese patients. Anticancer Res. 2018;38(7):3907-3911.

33. Białkowska K, Marciniak W, Muszyńska M, et al. Association of zinc level and polymorphism in MMP-7 gene with prostate cancer in Polish population. PLoS One. 2018;13(7):e0201065.

34. Lee C, Jia Z, Rahmatpanah F, et al. Role of the adjacent stroma cells in prostate cancer development and progression: synergy between TGF- $\beta$ and IGF signaling. Biomed Res Int. 2014;2014:502093-8.

35. Dayer C, Stamenkovic I. Recruitment of matrix metalloproteinase- 9 (MMP-9) to the fibroblast cell surface by lysyl hydroxylase 3 (LH3) triggers transforming growth factor- $\beta$ (TGF- $\beta$ ) activation and fibroblast differentiation. J Biol Chem. 2015;290(22):13763-13778. 


\section{Supplementary materials}

Table SI PRISMA checklist

\begin{tabular}{|c|c|c|c|}
\hline Section/topic & $\#$ & Checklist item & $\begin{array}{l}\text { Reported } \\
\text { on page \# }\end{array}$ \\
\hline \multicolumn{4}{|l|}{ Title } \\
\hline Title & $\mathrm{I}$ & Identify the report as a systematic review, meta-analysis, or both. & 1 \\
\hline \multicolumn{4}{|l|}{ Abstract } \\
\hline Structured summary & 2 & $\begin{array}{l}\text { Provide a structured summary including, as applicable: background, objectives, data } \\
\text { sources, study-eligibility criteria, participants, interventions, study appraisal and synthesis } \\
\text { methods, results, limitations, conclusions and implications of key findings, systematic } \\
\text { review registration number. }\end{array}$ & $2-3$ \\
\hline \multicolumn{4}{|l|}{ Introduction } \\
\hline Rationale & 3 & Describe the rationale for the review in the context of what is already known. & 3 \\
\hline Objectives & 4 & $\begin{array}{l}\text { Provide an explicit statement of questions being addressed with reference to } \\
\text { participants, interventions, comparisons, outcomes, and study design (PICOS). }\end{array}$ & $3-4$ \\
\hline \multicolumn{4}{|c|}{ r } \\
\hline Protocol and registration & 5 & $\begin{array}{l}\text { Indicate if a review protocol exists, if and where it can be accessed (eg, web address), } \\
\text { and if available provide registration information, including registration number. }\end{array}$ & \\
\hline Eligibility criteria & 6 & $\begin{array}{l}\text { Specify study characteristics (eg, PICOS, length of follow-up) and report characteristics } \\
\text { (eg, years considered, language, publication status) used as criteria for eligibility, giving } \\
\text { rationale. }\end{array}$ & 4 \\
\hline Information sources & 7 & $\begin{array}{l}\text { Describe all information sources (eg, databases with dates of coverage, contact with } \\
\text { study authors to identify additional studies) in the search and date last searched. }\end{array}$ & 4 \\
\hline Search & 8 & $\begin{array}{l}\text { Present full electronic search strategy for at least one database, including any limits used, } \\
\text { such that it could be repeated. }\end{array}$ & 4 \\
\hline Study selection & 9 & $\begin{array}{l}\text { State the process for selecting studies (ie, screening, eligibility, included in systematic } \\
\text { review, and if applicable included in the meta-analysis). }\end{array}$ & 4 \\
\hline Data collection process & 10 & $\begin{array}{l}\text { Describe method of data extraction from reports (eg, piloted forms, independently, in } \\
\text { duplicate) and any processes for obtaining and confirming data from investigators. }\end{array}$ & 4 \\
\hline Data items & 11 & $\begin{array}{l}\text { List and define all variables for which data were sought (eg, PICOS, funding sources) and } \\
\text { any assumptions and simplifications made. }\end{array}$ & \\
\hline $\begin{array}{l}\text { Risk of bias in individual } \\
\text { studies }\end{array}$ & 12 & $\begin{array}{l}\text { Describe methods used for assessing risk of bias of individual studies (including } \\
\text { specification of whether this was done at the study or outcome level), and how this } \\
\text { information is to be used in any data synthesis. }\end{array}$ & \\
\hline Summary measures & 13 & State the principal summary measures (eg, risk ratio, difference in means). & 5 \\
\hline Synthesis of results & 14 & $\begin{array}{l}\text { Describe the methods of handling data and combining results of studies, if done, } \\
\text { including measures of consistency }\left(\mathrm{eg}, l^{2}\right) \text { for each meta-analysis. }\end{array}$ & 5 \\
\hline Risk of bias across studies & 15 & $\begin{array}{l}\text { Specify any assessment of risk of bias that may affect the cumulative evidence (eg, } \\
\text { publication bias, selective reporting within studies). }\end{array}$ & $5-6$ \\
\hline Additional analyses & 16 & $\begin{array}{l}\text { Describe methods of additional analyses (eg, sensitivity or subgroup analyses, meta- } \\
\text { regression) if done, indicating which were prespecified. }\end{array}$ & $5-6$ \\
\hline \multicolumn{4}{|c|}{ 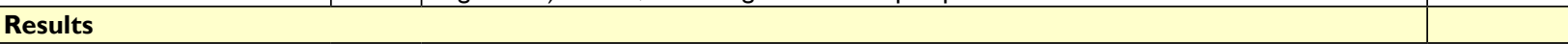 } \\
\hline Study selection & 17 & $\begin{array}{l}\text { Give numbers of studies screened, assessed for eligibility, and included in the review, } \\
\text { with reasons for exclusions at each stage, ideally with a flow diagram. }\end{array}$ & 6 \\
\hline Study characteristics & 18 & $\begin{array}{l}\text { For each study, present characteristics for which data were extracted (eg, study size, } \\
\text { PICOS, follow-up period) and provide the citations. }\end{array}$ & $6-7$ \\
\hline Risk of bias within studies & 19 & $\begin{array}{l}\text { Present data on risk of bias of each study, and if available any outcome-level assessment } \\
\text { (see item I2). }\end{array}$ & \\
\hline $\begin{array}{l}\text { Results of individual } \\
\text { studies }\end{array}$ & 20 & $\begin{array}{l}\text { For all outcomes considered (benefits or harms), present, for each study: (a) simple } \\
\text { summary data for each intervention group; (b) effect estimates and confidence intervals, } \\
\text { ideally with a forest plot. }\end{array}$ & 7 \\
\hline Synthesis of results & 21 & $\begin{array}{l}\text { Present results of each meta-analysis done, including confidence intervals and measures } \\
\text { of consistency. }\end{array}$ & 7 \\
\hline Risk of bias across studies & 22 & Present results of any assessment of risk of bias across studies (see item I5). & 8 \\
\hline Additional analysis & 23 & $\begin{array}{l}\text { Give results of additional analyses, if done (eg, sensitivity or subgroup analyses, meta- } \\
\text { regression [see item 16]). }\end{array}$ & 7 \\
\hline
\end{tabular}

(Continued) 
Table SI (Continued)

\begin{tabular}{|l|l|l|l|}
\hline Section/topic & $\#$ & Checklist item & $\begin{array}{l}\text { Reported } \\
\text { on page \# }\end{array}$ \\
\hline Discussion & 24 & $\begin{array}{l}\text { Summarize the main findings, including the strength of evidence for each main } \\
\text { outcome; consider their relevance to key groups (eg, health-care providers, users, and } \\
\text { policymakers). }\end{array}$ & 9 \\
\hline Summary of evidence & 25 & $\begin{array}{l}\text { Discuss limitations at study and outcome level (eg, risk of bias), and at review level (eg, } \\
\text { incomplete retrieval of identified research, reporting bias). }\end{array}$ & I0 \\
\hline Limitations & 26 & $\begin{array}{l}\text { Provide a general interpretation of the results in the context of other evidence, and } \\
\text { implications for future research. }\end{array}$ & II \\
\hline Conclusions & 27 & $\begin{array}{l}\text { Describe sources of funding for the systematic review and other support (eg, supply of } \\
\text { data); role of funders for the systematic review. }\end{array}$ & \\
\hline Funding &
\end{tabular}

Table S2 Quality-assessment scores

\begin{tabular}{|l|l|}
\hline Criteria & Score \\
\hline Representativeness of case & \\
Selected from population cancer registry & 2 \\
Selected from hospital & 1 \\
No method of selection described & 0 \\
Representativeness of control & \\
Population-based & 3 \\
Mixed & 2 \\
Hospital-based & 1 \\
Not described & 0 \\
Ascertainment of cancer case & \\
Histopathological confirmation & 2 \\
By patient medical record & 1 \\
Not described & 0 \\
Control selection & \\
Controls matched with cases by age and sex & 2 \\
Controls matched with cases only by age or by sex & 1 \\
Not matched or not described & 0 \\
Genotyping examination & \\
Genotyping done blindly and quality control & 2 \\
Only genotyping done blindly or quality control & 1 \\
Not described & 0 \\
HWE & \\
HWE in the control group & \\
HWD in the control group or not mentioned & 1 \\
Total sample size & 0 \\
$>I, 000$ & \\
$50 I-I, 000$ & 3 \\
$20 I-500$ & 2 \\
$\leq 200$ & 0 \\
\hline Abrial & \\
\hline
\end{tabular}

Abbreviations: HWE, Hardy-Weinberg equilibrium; HWD, HW disequilibrium. 
Table S3 Definition of comparison models

\begin{tabular}{|c|c|c|c|c|c|c|}
\hline MMP & SNP & Homozygote & Heterozygote & Dominant & Recessive & Additive \\
\hline MMPI & rsI799750 & IGIG vs $2 G 2 G$ & IG2G vs $2 G 2 G$ & IGIG+IG2G vs $2 \mathrm{G} 2 \mathrm{G}$ & IGIG vs IG2G+2G2G & IG vs $2 \mathrm{G}$ \\
\hline MMP2 & rs 243865 & CC vs TT & CT vs TT & $\mathrm{CC}+\mathrm{CT}$ vs TT & CC vs CT+TT & C vs T \\
\hline MMP2 & rs2285053 & CC vs TT & CT vs TT & $\mathrm{CC}+\mathrm{CT}$ vs TT & $\mathrm{CC}$ vs $\mathrm{CT}+\mathrm{TT}$ & C vs T \\
\hline MMP2 & rs 1477017 & $A A$ vs $G G$ & AG vs GG & $A A+A G$ vs $G G$ & $A A$ vs $A G+G G$ & A vs $G$ \\
\hline MMP2 & rs 17301608 & CC vs TT & CT vs TT & $\mathrm{CC}+\mathrm{CT}$ vs TT & $\mathrm{CC}$ vs $\mathrm{CT}+\mathrm{TT}$ & C vs T \\
\hline MMP2 & rsII639960 & $A A$ vs GG & AG vs GG & $\mathrm{AA}+\mathrm{AG}$ vs $\mathrm{GG}$ & $A A$ vs $A G+G G$ & A vs $G$ \\
\hline MMP3 & $1171-5 \mathrm{~A} / 6 \mathrm{~A}$ & $5 \mathrm{~A} 5 \mathrm{~A}$ vs $6 \mathrm{~A} 6 \mathrm{~A}$ & $5 \mathrm{~A} 6 \mathrm{~A}$ vs $6 \mathrm{~A} 6 \mathrm{~A}$ & $5 A 5 A+5 A 6 A$ vs $6 A 6 A$ & $5 \mathrm{~A} 5 \mathrm{~A}$ vs $5 \mathrm{~A} 6 \mathrm{~A}+6 \mathrm{~A} 6 \mathrm{~A}$ & $5 \mathrm{~A}$ vs $6 \mathrm{~A}$ \\
\hline MMP3 & $1161-A / G$ & $A A$ vs $G G$ & AG vs GG & $A A+A G$ vs $G G$ & $A A$ vs $A G+G G$ & $A$ vs $G$ \\
\hline MMP3 & $5356-\mathrm{A} / \mathrm{G}$ & $A A$ vs $G G$ & AG vs GG & $A A+A G$ vs $G G$ & $A A$ vs $A G+G G$ & A vs $G$ \\
\hline MMP7 & rsII5688|8 & $A A$ vs $G G$ & AG vs GG & $A A+A G$ vs $G G$ & $A A$ vs $A G+G G$ & $A$ vs $G$ \\
\hline MMP9 & rs 17576 & $A A$ vs $G G$ & AG vs GG & $A A+A G$ vs $G G$ & $A A$ vs $A G+G G$ & $A$ vs $G$ \\
\hline MMPI3 & rs2252070 & TT vs CC & TC vs CC & $\mathrm{TT}+\mathrm{TC}$ vs $\mathrm{CC}$ & TT vs TC $+\mathrm{CC}$ & T vs $C$ \\
\hline
\end{tabular}

Table S4 Frequency of genotype in studies from meta-analysis. (A) MMPI rs I799750; (B) MMP2 rs243865; (C) MMP7 rs I I5688I8

\begin{tabular}{|c|c|c|c|c|c|c|c|c|}
\hline \multicolumn{9}{|l|}{ A } \\
\hline \multirow[t]{2}{*}{ First author } & \multirow[t]{2}{*}{ MMP } & \multirow[t]{2}{*}{ SNP } & \multicolumn{3}{|l|}{ Case } & \multicolumn{3}{|c|}{ Control } \\
\hline & & & IGIG & IG2G & $2 G 2 G$ & IGIG & IG2G & 2G2G \\
\hline Albayrak $S^{\prime}$ & MMPI & rsI799750 & 10 & 7 & 38 & 7 & 3 & 33 \\
\hline Dos Reis $\mathrm{ST}^{2}$ & MMPI & rsI799750 & 21 & 52 & 27 & 11 & 34 & 55 \\
\hline Tsuchiya $\mathrm{N}^{3}$ & MMPI & rs 1799750 & 35 & 122 & 126 & 33 & 100 & 118 \\
\hline Liao $\mathrm{CH}^{4}$ & MMPI & rsI799750 & 51 & 88 & 79 & 96 & 193 & 147 \\
\hline Białkowska $\mathrm{K}^{5}$ & MMPI & rsI799750 & 56 & 105 & 36 & 54 & 90 & 53 \\
\hline \multicolumn{9}{|l|}{ B } \\
\hline \multirow[t]{2}{*}{ First author } & \multirow[t]{2}{*}{ MMP } & \multirow[t]{2}{*}{ SNP } & \multicolumn{3}{|l|}{ Case } & \multicolumn{3}{|c|}{ Control } \\
\hline & & & CC & CT & TT & CC & CT & TT \\
\hline Dos Reis $\mathrm{ST}^{2}$ & MMP2 & rs243865 & 50 & 38 & 12 & 59 & 20 & 21 \\
\hline Srivastava $\mathrm{P}^{6}$ & MMP2 & rs 243865 & 101 & 78 & 11 & 131 & 62 & 7 \\
\hline Yaykasli KO ${ }^{7}$ & MMP2 & rs243865 & 51 & 7 & 3 & 42 & 4 & 0 \\
\hline Adabi $Z^{8}$ & MMP2 & rs 243865 & 74 & 27 & 0 & 113 & 23 & $\mathrm{I}$ \\
\hline Shajarehpoor Salavati L ${ }^{9}$ & MMP2 & rs243865 & 34 & 11 & 5 & 41 & 7 & 6 \\
\hline Białkowska $\mathrm{K}^{5}$ & MMP2 & rs 243865 & 104 & 79 & 14 & 101 & 78 & 18 \\
\hline \multicolumn{9}{|l|}{ C } \\
\hline \multirow[t]{2}{*}{ First author } & \multirow[t]{2}{*}{ MMP } & \multirow[t]{2}{*}{ SNP } & \multicolumn{3}{|l|}{ Case } & \multicolumn{3}{|c|}{ Control } \\
\hline & & & $\mathbf{A A}$ & AG & GG & AA & AG & GG \\
\hline Dos Reis $\mathrm{ST}^{2}$ & MMP7 & rsII5688I8 & 33 & 41 & 26 & 25 & 39 & 36 \\
\hline Białkowska $\mathrm{K}^{5}$ & MMP7 & $\mathrm{rs}|| 5688 \mid 8$ & 59 & 100 & 38 & 76 & 97 & 24 \\
\hline
\end{tabular}


Table S5 Frequency of genotype in studies from systematic review

\begin{tabular}{|c|c|c|c|c|c|c|c|c|}
\hline \multirow{3}{*}{$\begin{array}{l}\text { First author } \\
\text { Srivastava } \mathrm{P}^{6}\end{array}$} & \multirow{3}{*}{$\begin{array}{l}\text { MMP } \\
\text { MMP2 }\end{array}$} & \multirow{3}{*}{\begin{tabular}{|l|} 
SNP \\
rs2285053 \\
\end{tabular}} & \multicolumn{3}{|l|}{ Case } & \multicolumn{3}{|c|}{ Control } \\
\hline & & & $\mathrm{CC}$ & $\mathrm{CT}$ & TT & CC & CT & TT \\
\hline & & & 101 & 78 & 11 & 131 & 62 & \begin{tabular}{|l|}
7 \\
\end{tabular} \\
\hline \multirow[t]{2}{*}{ Jacobs EJ'10 } & \multirow[t]{2}{*}{ MMP2 } & \multirow{2}{*}{ rs I4770I7 } & AA & AG & GG & AA & AG & GG \\
\hline & & & 566 & 645 & 206 & 639 & 624 & 178 \\
\hline \multirow{2}{*}{ Jacobs EJ'10 } & \multirow{2}{*}{ MMP2 } & \multirow[t]{2}{*}{ rsI730I608 } & $\mathrm{CC}$ & $\mathrm{CT}$ & TT & $\mathrm{CC}$ & $\mathrm{CT}$ & TT \\
\hline & & & 541 & 655 & 218 & 600 & 650 & 182 \\
\hline \multirow[t]{2}{*}{ Jacobs EJ'10 } & \multirow[t]{2}{*}{ MMP2 } & \multirow[t]{2}{*}{ rsII639960 } & AA & AG & GG & AA & AG & GG \\
\hline & & & 597 & 645 & 168 & 675 & 610 & 154 \\
\hline \multirow[t]{2}{*}{ Srivastava $\mathrm{P}^{\prime \prime}$} & \multirow[t]{2}{*}{ MMP3 } & \multirow[t]{2}{*}{$1 I 7 I-5 A / 6 A$} & $5 A 5 A$ & $5 \mathrm{~A} 6 \mathrm{~A}$ & 6A6A & 5A5A & $5 \mathrm{~A} 6 \mathrm{~A}$ & 6A6A \\
\hline & & & 11 & 38 & 101 & 4 & 64 & 132 \\
\hline \multirow[t]{2}{*}{ Srivastava $\mathrm{P}^{\prime \prime}$} & \multirow[t]{2}{*}{ MMP3 } & \multirow[t]{2}{*}{$I I 6 I-A / G$} & AA & $A G$ & GG & AA & $A G$ & GG \\
\hline & & & 77 & 66 & 7 & 103 & 80 & 17 \\
\hline \multirow[t]{2}{*}{ Srivastava PII } & \multirow[t]{2}{*}{ MMP3 } & \multirow[t]{2}{*}{ 5356-A/G } & AA & AG & GG & $\mathrm{AA}$ & AG & GG \\
\hline & & & 54 & 84 & 12 & 84 & 89 & 27 \\
\hline \multirow[t]{2}{*}{ Dos Reis $\mathrm{ST}^{2}$} & \multirow[t]{2}{*}{ MMP9 } & \multirow[t]{2}{*}{ rs I7576 } & AA & AG & GG & AA & AG & GG \\
\hline & & & $\mathrm{I}$ & 43 & 56 & 5 & 93 & 2 \\
\hline \multirow[t]{2}{*}{ Białkowska $\mathrm{K}^{5}$} & \multirow[t]{2}{*}{ MMPI3 } & \multirow[t]{2}{*}{ rs2252070 } & TT & $\mathrm{CT}$ & CC & TT & CT & CC \\
\hline & & & 92 & 87 & 18 & 104 & 78 & 15 \\
\hline
\end{tabular}

\section{References}

1. Albayrak S, Cangüven O, Göktaş C, Aydemir H, Köksal V. Role of MMP-1 1G/2G promoter gene polymorphism on the development of prostate cancer in the Turkish population. Urol Int. 2007;79(4):312-315.

2. Dos Reis ST, Pontes J Jr, Villanova FE, et al. Genetic polymorphisms of matrix metalloproteinases: susceptibility and prognostic implications for prostate cancer. $J$ Urol. 2009;181(5):2320-2325.

3. Tsuchiya N, Narita S, Kumazawa T, et al. Clinical significance of a single nucleotide polymorphism and allelic imbalance of matrix metalloproteinase-1 promoter region in prostate cancer. Oncol Rep. 2009;22(3):493-439.

4. Liao CH, Wu HC, Hu PS, et al. The association of matrix metalloproteinase-1 Promoter polymorphisms with prostate cancer in taiwanese patients. Anticancer Res. 2018;38(7):3907-3911.

5. Białkowska K, Marciniak W, Muszyńska M, et al. Association of zinc level and polymorphism in MMP-7 gene with prostate cancer in Polish population. PLoS One. 2018;13(7):e0201065.
6. Srivastava P, Lone TA, Kapoor R, Mittal RD. Association of promoter polymorphisms in MMP2 and TIMP2 with prostate cancer susceptibility in North India. Arch Med Res. 2012;43(2):117-1124.

7. Yaykaşli KO, Kayikçi MA, Yamak N, et al. Polymorphisms in MMP-2 and TIMP-2 in Turkish patients with prostate cancer. Turk $J$ Med Sci. 2014;44(5):839-843.

8. Adabi Z, Mohsen Ziaei SA, Imani M, et al. Genetic polymorphism of MMP2 gene and susceptibility to prostate cancer. Arch Med Res. 2015;46(7):546-550.

9. Shajarehpoor Salavati L, Tafvizi F, Manjili HK.The association between MMP2 -1306 C > T (rs243865) polymorphism and risk of prostate cancer. Ir J Med Sci. 2017;186(1):103-111.

10. Jacobs EJ, Hsing AW, Bain EB, et al. Polymorphisms in angiogenesisrelated genes and prostate cancer. Cancer Epidemiol Biomarkers Prev. 2008;17(4):972-977.

11. Srivastava P, Kapoor R, Mittal RD. Impact of MMP-3 and TIMP-3 gene polymorphisms on prostate cancer susceptibility in North Indian cohort. Gene. 2013;530(2):273-277.
Cancer Management and Research

\section{Publish your work in this journal}

Cancer Management and Research is an international, peer-reviewed open access journal focusing on cancer research and the optimal use of preventative and integrated treatment interventions to achieve improved outcomes, enhanced survival and quality of life for the cancer patient. The manuscript management system is completely online and includes

\section{Dovepress}

a very quick and fair peer-review system, which is all easy to use. Visit $\mathrm{http}: / / \mathrm{www}$.dovepress.com/testimonials.php to read real quotes from published authors. 